\title{
Alteraciones en el gasto energético en reposo y parámetros respiratorios en pacientes con síndrome de Prader-Willi
}

\author{
Abnormalities in energy expenditure and \\ respiratory parameters in patients \\ with Prader Willy syndrome
}

\begin{abstract}
Introduction: Prader Willi Syndrome (PWS) is a complex genetic disorder with many manifestations relating to hypothalamic insufficiency. One such feature is obesity associated with an imbalance between caloric intake and expenditure due to hyperphagia and slow metabolic rate. Different procedures, as the use of appetite suppressants, surgical interventions and change in behavioral techniques have been used to try to limit food intake, but there is not convincing results about it. Aim: To compare the resting energy expenditure (REE) in PWS patients with obese patients without the syndrome matched by age and BMI (Body Mass Index). Subjects and methods: We conducted a comparative, observational and descriptive study in PWS and obese patients, matched for age, sex and BMI. RER was measured by indirect calorimerty and by anthropometry (BMI, skin-fold triceps and bíceps). A t-Student test for independent samples was used to demonstrate homogeneity between subjects and a series of ANCOVA analysis was performed on each of the parameters displayed by the indirect calorimetry. Results: Twenty mestizo-mexican patients, 10 with PWS and 10 with obesity were analyzed matched by age, BMI and gender. We found a statistically significant difference in the REE, the volume of oxygen $(\mathrm{ml} / \mathrm{min})$ and tidal volume $(p<0.05)$. Conclusions: Prader Willy Syndrome per se produces changes in the REE in comparison with patients with only obesity.
\end{abstract}

Key words: Prader-Willi syndrome, obesity, resting energy expenditure.

\section{INTRODUCCION}

El Síndrome de Prader Willi (SPW) constituye la causa más frecuente de obesidad de origen genético (1). Se estima que la incidencia es 1/10,0002,3 a 1/15,000, presentándose en ambos sexos y en todos los grupos étnicos. El síndrome se caracteriza por obesidad, hipotonía severa al nacimiento, talla baja, hipogonadismo y retraso psicomotor (1-6). Estos pacientes tienen alto riesgo de presentar complicaciones metabólicas relacionadas con la obesidad como diabetes mellitus de aparición temprana y datos de Síndrome metabólico, lo que incrementa la morbi-mortalidad (5,7-9).

El SPW se considera, una de las afecciones genéticas y uno de los síndromes de microdeleción más frecuentes, causado por una pérdida de expresión del alelo paterno en
Vanessa Fuchs-Tarlovsky (1) Karolina Alvarez A. (1)

Evelyn Sifuentes G. $(1,2)$

Liliana Aleman G. $(1,2)$

Marene Parra B. $(1,2)$

Gloria Queipo G. (2-3)

(1) Servicio de Ontología, Hospital General de México, México (2) Servicio de Genética Hospital General de México, México (3) Facultad de Medicina Universidad Nacional Autónoma de México.

Dirigir la correspondencia a: Profesora Gloria Queipo G Departamento de Genética Humana Hospital General de México Facultad de Medicina, Universidad Nacional Autónoma de México México DF Dr. Balmis $146 \mathrm{Col}$, Doctores CP 06726 Mexico DF. E-mail: gqueipo99@yahoo.com: gqueipo99@exalumno.unam.mx

Este trabajo fue recibido el 19 de Junio de 2012 y aceptado para ser publicado el 28 de Octubre de 2012. un grupo de genes improntados localizados en una región de $2-M b$ en $15 q 11-q 13(10,11)$. Aproximadamente $75 \%$ de los individuos tienen una deleción paterna en ésta región; el $24 \%$ de los casos se debe a una disomía uniparental materna y el resto es ocasionado por alteraciones en el centro improntador (12,13).

La obesidad es resultado de anormalidades hipotalámicas, principalmente hiperfagia, la cual inicia entre el primer y sexto año de vida $(14,15)$, y está condicionada por anomalías hipotalámicas con fallas en el mecanismo de retroalimentación que normalmente llevaría a la saciedad $(16,17)$.

Los pacientes con PWS tienen una baja tasa metabólica y por lo tanto un requerimiento calórico muy bajo (6). Es un desafío el prevenir el desarrollo de obesidad utilizando dietas 
con suficiente aporte energético en nutrientes esenciales que aseguren un crecimiento óptimo (18). La expectativa de vida en personas con PWS esta relacionada con el control de peso (19).

Modificación en las técnicas de comportamiento e intervenciones quirúrgicas han sido utilizadas para tratar de limitar el consumo de alimentos, pero no hay resultados contundentes al respecto. En el presente no hay tratamiento específico a la falla de saciedad y comportamiento de sobrealimentación (20). En estos niños se han reportado niveles bajos de adiponectina secundario a las alteraciones en la secreción de insulina (21). El consumo nutricional para niños pequeños en desarrollo y crecimiento es un tema importante $(22,23)$. Es importante conocer el comportamiento metabólico de los pacientes con SPW para establecer una ingesta dietética y un estilo de vida de acuerdo al la tasa metabólica real además de la terapia con hormona del crecimiento. Hay pocos estudios en la literatura sobre el comportamiento metabólico en pacientes con obesidad debido a este síndrome.

El presente trabajo analizó de forma sistemática el gasto energético en reposo (GER) en un grupo de 10 pacientes con SPW y se parearon con pacientes obesos sin el síndrome.

\section{SUJETOS Y METODO}

Se realizó un estudio comparativo observacional, transversal y descriptivo, se compararon un grupo de 10 pacientes con SPW contra niños sin SPW pareados por edad, sexo e IMC.

El estudio fue aprobado por los comités de Bioética e investigación del Hospital General de México. Los padres o tutores de los participantes firmaron una carta de consentimiento informado. Los pacientes con SPW fueron diagnosticados mediante el estudio de metilación alelo específica del gen SNRP. En todos los sujetos de estudio así como en los controles se realizó una evaluación antropométrica y una calorimetría indirecta a fin de conocer la composición corporal y el gasto energético en reposo (GER). La evaluación antropométrica consistió en medir el peso, la talla actuales así como medidas antropométricas (pliegue cutáneo tricipital y pliegue cutáneo bicipital) usando un plicómetro de Lange con previa estandarización con método de Habitch (18). El cálculo del gasto energético en reposo por calorimetría indirecta se realizó tras 8 horas de ayuno y sin haber realizado actividad física previa, utilizando un calorímetro REEVUE (Korres $\left.{ }^{T M}\right)$. Se registró el coeficiente respiratorio (QR) así como el GER.

La comparación estadística entre los sujetos de estudio se realizó usando el programa de análisis estadístico SPSS V.17 para Windows. Para identificar la homogeneidad de los grupos respecto su IMC y edad se utilizó una prueba T de Student para muestras independientes. Se realizó una serie de análisis de Ancova sobre cada uno de los parámetros de la calorimetría indirecta realizada a casos y controles. Se tomaron como variables independientes todos los parámetros de la calorimetría, como variable dependientes se tomaron grupo y sexo, y como covariables edad e IMC, ajustadas en 28.35 $\mathrm{kg} / \mathrm{m}^{2}$ y 9.05 años respectivamente.

\section{RESULTADOS}

La población estudiada incluyó un total de 20 pacientes mestizo-mexicanos: 10 pacientes con SPW y 10 controles obesos, las características antropométricas y parámetros clínicos se observan en la tabla 1. De 10 pacientes con SPW, $6(60 \%)$ eran mujeres y $4(40 \%)$ varones, con un promedio de edad de $9 \pm 7.2$ años y un IMC de $28.64 \pm 10.8 \mathrm{~kg} / \mathrm{m}^{2}$. El grupo de controles obesos se encontraba distribuido en 7 (70\%) mujeres y 3 (30\%) varones con un promedio de edad $9.1 \pm 7.5$ años y un IMC de $28.06 \pm 6.25 \mathrm{~kg} / \mathrm{m}^{2}$. Esto muestra que ambos grupos son homogéneos en estos parámetros y que todas las diferencias observadas podrían ser atribuidas a las características del síndrome y no a la composición corporal o la obesidad per se.

En la tabla 2 se observa la comparación de los resultados obtenidos de la calorimetría indirecta. Se identificó una diferencia estadísticamente significativa en el requerimiento energético en reposo (GER). El estudio calorimétrico incluye en su análisis final el estudio de varios marcadores de función respiratoria fueron analizados con el objetivo de ver si alguno de estos tenían diferencias. Se observaron diferencias significativas en el volumen de oxígeno ( $\mathrm{mil} / \mathrm{min}$ ) y volumen TIDAL (figura I A-C) ( $p$ <0.05). Lo que se explica como cambios secundarios a la suma del síndrome y la obesidad, situación que no se observa en los controles que tienen como condición patológica únicamente la obesidad.

\section{DISCUSIÓN}

El SPW es la causa mas frecuente de obesidad de origen genético, estos pacientes tienen alto riesgo de presentar complicaciones metabólicas relacionadas con la ganancia de peso como la aparición temprana de diabetes mellitus y el síndrome metabólico. Las características clínicas más relevantes que participan en el desarrollo de las complicaciones metabólicas en estos niños son la hipotonía severa que se observa desde el nacimiento, la hiperfagia que se inicia desde la infancia, y el difícil control alimentario; todo esto secundario a las alteraciones en el desarrollo hipotalámico propio del síndrome.

TABLA 1

Características clínicas y antropométricas en pacientes con SPW.

\begin{tabular}{lccccccccccc}
\hline Características & PW 1 & PW 2 & PW 3 & PW 4 & PW 5 & PW 6 & PW 7 & PW 8 & PW 9 & PW 10 \\
Sexo & & F & F & $M$ & F & M & M & F & F & F & M \\
Edad (años) & 2 & 4 & 5 & 6 & 7 & 7 & 8 & 8 & 17 & 26 \\
IMC (Kg/mt2) & 19.2 & 14.8 & 19.2 & 26 & 26 & 30 & 36.1 & 26 & 37.1 & 52 \\
PCT (cm) & 10 & 12 & 12 & 17 & 23 & 25 & 28 & 21 & 41 & 54 \\
PCB (cm) & 10 & 9 & 12 & 13 & 20 & 14 & 19 & 16 & 22 & 39 \\
\hline
\end{tabular}

SPW $=$ Síndrome de Prader-Willi.

$\mathrm{PCT}=$ Pliegue cutáneo de triceps.

$\mathrm{PCB}=$ Pliegue cutáneo de bíseps.

$I M C=$ índice de masa corporal 
En la literatura no existen suficientes reportes que estudien cual es el comportamiento en GER, y parámetros respiratorios en pacientes con SPW comparados pacientes obesos. Uno de los primeros estudios formales donde se midió la composición corporal, el gasto energético total y en reposo en un grupo de pacientes con PWS indicó que el grupo de pacientes con el síndrome tenían una disminución en el GER 7,23. Sin embargo, no existen otros reportes contundentes en

TABLA 2

Media marginal estimada de calorimetría indirecta en pacientes con SPW y sin el síndrome.

\begin{tabular}{|c|c|c|c|c|}
\hline \multirow[b]{2}{*}{ Valores de calorimetría } & \multicolumn{2}{|c|}{ Prader-Willi } & \multicolumn{2}{|c|}{ Obesos } \\
\hline & Media & Error estándar & Media & Error estándar \\
\hline GER* & 1096.762 & 50.636 & 1331.131 & 52.777 \\
\hline VO2MIMin* & 161.7 & 8.165 & 213.139 & 8.51 \\
\hline VO2MIMinK & 4.85 & 0.498 & 4.859 & 0.519 \\
\hline $\mathrm{FeO} 2$ & 16.373 & 0.258 & 15.97 & 0.269 \\
\hline TIDALVol* & 0.022 & 0.115 & 0.64 & 0.12 \\
\hline RspRateBPM* & 31.575 & 3.706 & 19.748 & 3.863 \\
\hline $\begin{array}{l}\text { * } p<0.05, \text { ANOVA corregida por } \\
\text { SPW }=\text { Síndrome de Prader-Willi. } \\
\text { GER }=\text { Gasto energético en reposo. } \\
\text { VO2MIMin=Volumen de oxígeno } \\
\text { VO2MIMinK= Volumen de oxígen } \\
\text { FEO2 = Fracción de oxígeno espira } \\
\text { TIDALVol= Volumen TIDAL. } \\
\text { RspRateBPM= Respiraciones por }\end{array}$ & ños) e IMC (28. & ntre pacientes con SP & obesos. & \\
\hline
\end{tabular}

\section{FIGURA 1}

Diferencias estadísticas en (A) Gasto Energético en Reposo (GER), (B) Volumen TIDAL y (C) Volumen de oxígeno en los parámetros de la calorimetría indirecta realizada entre los pacientes con SPW y controles obesos.

A

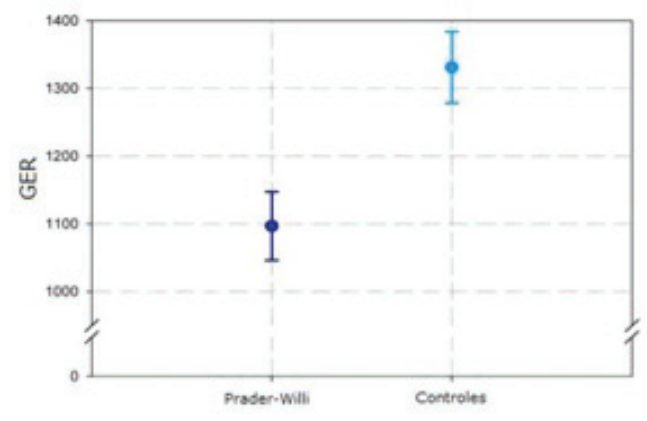

B

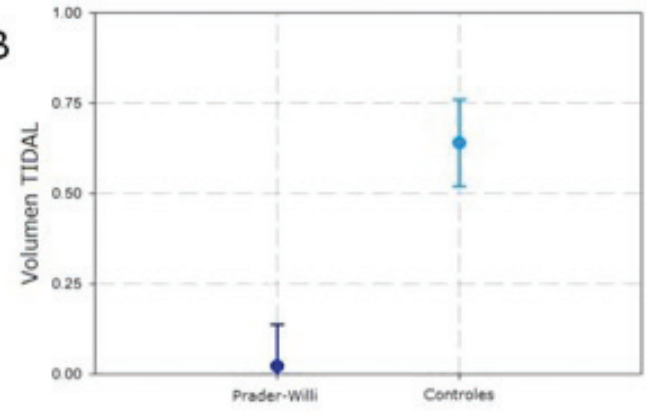

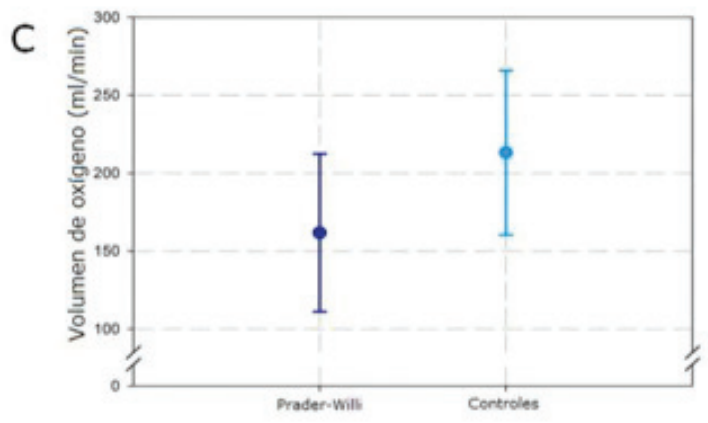


la literatura que confirmen este hallazgo. Por lo que utilizando la calorimetría indirecta, método moderno, sensible y específico para determinar el gasto energético de determinadas poblaciones realizamos este estudio con los pacientes de PWS que acuden a la clínica del Servicio de Genética del Hospital General de México.

Las características antropométricas de los pacientes con el síndrome muestran que 7/10 pacientes fueron obesos mientras que el resto no. En estos 3/10 pacientes, la terapia temprana con hormona del crecimiento podría explicar la falta de obesidad. Los resultados de la calorimetría indirecta obtenida en nuestro estudio confirman los resultados previos ya que se observó una disminución estadísticamente significativa en el GER. El grupo de pacientes con SPW tuvo un gasto energético en reposo $17.67 \%$ menor al grupo de pacientes obesos. Estos resultados son muy similares a los reportados por Butler y colaboradores en el 2007, donde utilizando un sofisticado sistema de medición con absorciometría dual por rayos-X (DEXA) y la cuantificación de $\mathrm{CO} 2$ en una cámara metabólica identificando en los pacientes con Prader Willi una disminución del 16\% en el GER (24). Los resultados son interesantes ya que utilizando diferentes sistemas de medición más accesibles y de menor costo, así como un número menor de pacientes los resultados son similares; la homogeneidad por edad e IMC en nuestros grupos de estudio nos permite concluir que las alteraciones en GER observadas en los pacientes son secundarias al síndrome y no al elevado IMC.

Otro dato interesante fue las diferencias observadas en el volumen de oxígeno ( $\mathrm{ml} / \mathrm{min}$ ) y el volumen TIDAL, ya que ambos fueron estadísticamente menores en el grupo de pacientes con SPW, comparado con los controles $(p<0.05)$. La probable explicación a esto es que el patrón restrictivo pulmonar se estableció en etapas más tempranas de la vida, ya que la obesidad en los pacientes con SPW se inicia en etapas tempranas comparada con el grupo de pacientes obesos en los cuales la obesidad se establece después. Otra condición importante en nuestro grupo experimental fue la presencia de hipotonía lo que condicionaba que los pacientes tuvieran un patrón respiratorio anormal desde el nacimiento. Todos los pacientes reportaron diferentes formas de apneas del sueño verificadas por polisomnografía del sueño (datos no mostrados). Aunque la muestra no es representativa la utilización de hormona de crecimiento modificó los parámetros en los 3 niños que no presentaron obesidad y sus parámetros respiratorios mejoraron. Publicaciones anteriores han demostrado que la hormona de crecimiento mejora la condición pulmonar de los pacientes con SPW (25). Otras condiciones que se agregan a estos hallazgos son la falta de actividad física, el difícil control dietético y las alteraciones cognitivas que tienen los pacientes con el síndrome comparados con los sujetos obesos sin el síndrome.

El presente trabajo deja claro que las modificaciones en el GER son una característica propia del SPW y que esta condición coadyuva con las demás características en el daño metabólico que se va desarrollando a lo largo de la vida del individuo. Aunque no fue el objetivo del trabajo se observó que el tratamiento con hormona de crecimiento mejora estas condiciones. El tratamiento esta indicado desde etapas tempranas disminuyendo considerablemente las co-morbilidades propias del síndrome. Este estudio también confirmó los datos ya conocidos sobre las alteraciones en la función pulmonar de los pacientes. Con todo lo anterior concluimos que es importante realizar el diagnóstico lo más temprano posible, con la finalidad de poder iniciar un manejo multidisciplinario temprano y prolongar la aparición de las complicaciones de los pacientes con el síndrome.

\section{RESUMEN}

Introducción: El síndrome de Prader Willi (SPW) es un trastorno genético complejo relacionado con insuficiencia hipotalámica. Una de las manifestaciones clínicas es la obesidad asociada a un desequilibrio entre la ingesta y el gasto energético, debido a la hiperfagia y a la baja tasa metabólica que presentan estos pacientes. Distintos procedimientos e intervenciones han sido utilizadas para tratar de limitar el consumo de alimentos, pero no hay resultados contundentes al respecto. Objetivo: Comparar el gasto energético en reposo (GER) de pacientes con SPW con pacientes obesos sin el Síndrome. Sujetos y métodos: Se realizó un estudio comparativo observacional, transversal y descriptivo en pacientes con SPW y pacientes obesos sin SPW pareados por edad, sexo e IMC. Se midió por medio de calorimetría indirecta el GER de los pacientes, así como por antropometría, IMC, pliegue cutáneo de tríceps y bíceps. Se utilizó una prueba T de Student para muestras independientes que comprobara la homogeneidad de los pacientes y se realizó una serie de análisis de ANCOVA sobre cada uno de los parámetros de la calorimetría indirecta. Resultados: Se analizó 20 pacientes mestizo-mexicanos entre 2 y 26 años: 10 pacientes con SPW y 10 controles obesos. Se identificó una diferencia estadísticamente significativa en el GER, resultando $17.67 \%$ menor en los sujetos con SPW; así también en el volumen de oxígeno ( $\mathrm{mil} / \mathrm{min}$ ) y volumen TIDAL $(p<0.05)$. Conclusión: Pacientes con SPW per se producen modificaciones en el GER a lo largo de la vida en comparación con los pacientes con obesidad.

Palabras clave: Síndrome de Prader Willi, obesidad, gasto energético en reposo.

Agradecimientos: Este trabajo se realizó en el Servicio de Genética Humana del Hospital General de México-Facultad de Medicina UNAM. El apoyo fue otorgado por la Dirección de Investigación del Hospital General de México, y el CONACYT en su "grant" número 115440.

\section{BIBLIOGRAFÍA}

1. Burd L, Vesely B, Martsolf J, Kerbeshian J. 1990. Prevalence study of Prader-Willy syndrome in North Dakota. Am J Med Genet 37:97-9.

2. Butler MG. 1990. Prader-Willi syndrome: current understanding of cause and diagnosis. Am J Med Genet 35:319-32.

3. Butler MG, Thompson T. 2000. Prader-Willy syndrome: clinical and genetic finding. Endocrinology 10, 3S-16S.

4. Bittel DC, Butler MG. 2005. Prader-Willy syndrome: Clinical genetics, cytogenetics and molecular biology. Expert Rev Mol Med 7(14):1-20.

5. Solà J, Giménez G. 2006. Abordaje integral del syndrome de Prader-Willi en la edad adulta. Endo Nutr 53(3):181-9.

6. Cassidy SB, Driscoll DJ. 2009. Prader-Willy syndrome. Eur J Hum Genet 17(1):3-13

7. Van EA Westerterp KR Gerver W] Curfs LM, SchranderStumpel CA Kester AD Saris WH 2000. Energy expenditure at rest and during sleep in children with Prader-Willy syndrome is explained by body composition. Am J Clin Nutr 71(3):752-6.

8. Butler JV, Whittington JE, Holland AJ, Boer H, Clarke D, Webb T. 2002. Prevalence of, and risk factors for, physical ill-health in people with Prader-Willy syndrome: a pop- 
ulation-based study. Rev Med Child Neurol 44:248- 55.

9. Butler MG. 2011 Prader-Willy Syndrome: Obesity due to Genomic Imprinting. Cur Gen 12:204-15.

10. Ledbetter DH, Riccardi VM, Airhart SD, Strobel RJ, Keenan BS, Crawford JD. 1981. Deletions of chromosome 15 as a cause of the Prader-Willy syndrome. N Engl J Med 304:325-9.

11. Butler MG, Palmar CG. 1983 Parental origin of chromosome 15 deletion in Prader-Willy syndrome. Lancet 1983 I:285-6.

12. Amos JM, Ji Y, Gottlieb W, Depinet $T$, Wandstrat $A E$, Cassidy SB, Driscoll DJ, Rogan PK, Schwartz S, Nicholls RD. 1999. Chromosome breakage in the Prader-Willy and Angelman syndromes involves recombination between large, transcribed repeats at proximal and distal breakpoints. Am J Hum Genet 65:370-86.

13. Chai JH, Locke DP, Greally JM, Knoll JH, Ohta T, Dunai J, Yavor A, Eichler EE, Nicholls RD. 2003. Identification of four highly conserved genes between breakpoint hotspots BP1 and BP2 of the Prader-Willy/Angelman syndromes deletion region that have undergone evolutionary transposition mediated by flanking duplicons. Am J Hum Genet 73:898-925

14. Zipf WB, Berntson GG. 1987. Characteristics of abnormal food-intake patterns in children with Prader-Willy syndrome and study of effects of naloxone. Am J Clin Nutr 46:277-81.

15. Swaab DF, Purba JS, Hofman MA. 1995. Alterations in the hypothalamic paraventricular nucleus and its oxytocin neurons (putative satiety cells) in Prader-Willy syndrome: a study of five cases. J Clin Endocrinol Metab 80:573-9.

16. Fieldstone A, Zipf WB, Sarter MF, Bertson GG. 1988. Food intake in Prader-Willy syndrome and controls with obesity after administrationof benzodiazepine receptor agonist. Obes Res 6:29-33.

17. Holland AJ, Treasure J, Coskeran P, Dallow J, Milton
$N$, Hillhouse E. Measurement of excessive appetite and metabolic changes in Prader-Willi syndrome. Int J Obes 1993; 17:527-32.

18. Santos VM, Henrique de Paula F, Osterne EM, Nery NS, Turra Tz. 2009. Morbid obesity in an adolescent with Prader-Willi syndrome. Rev Med Chil 137: 264-8.

19. Williams $K$, Scheimann A, Sutton V, Hayslett E, Glaze $D$. Sleepiness and sleep disordered breathing in PraderWilly syndrome: relationship to genotype, growth hormone therapy, and body composition, J Clin Sleep Med 2008; 4(2): 111-8.

20. Braghetto I, Rodríguez A, Debandi L, Brunet L, Papapietro K. 2003. Síndrome Prader-Willi asociado a obesidad mórbida: tratamiento quirúrgico. Rev Med Chil 131:427-31.

21. Haqq AM, Muehlbauer M, Svetkey $L P$, Newgard CB, Purnell JQ, Grambow SC, Freemark MS. 2007. Altered distribution of adiponectin isoforms in children with Prader-Willy syndrome (PWS): association with insulin sensitivity and circulating satiety peptide hormones. Clin Endocrinol (Oxf) 67:944-51.

22. Modesto V, De Paula F, Misael E, Solón N, Zavascki T. 2009. Morbid obesity in an adolescent with Prader-Willy syndrome. Rev Med Chile 137: 264-8.

23. Lindmark M, Trygg K, Gilvedt K, Kolset SO. 2010. Nutrient intake of young children with Prader-Willy syndrome. Food Nutr Res 54:1-6.

24. Butler MG, Theodoro MF, Bittel DC, Donnelly JE. Energy Expenditure and Physical Activity in Prader-Willy Syndrome: Comparison With Obese Subjects. Am J Med Genet 2007; 143A:449-59.

25. Haqd $A M$, Stadlen $D D$, lackson RH, Rosenfeld RG, Purnell JQ and LaFranchi AM. 2003. Effects of Growth Hormone on Pulmonary Function, Sleep Quality, Behavior, Cognition, Growth Velocity, Body Composition, and Resting Energy Expenditure in Prader-Willy Syndrome. J Clin Endocrinol Metab 88(5):2206-12. 